\title{
Medical Image of the Week: Necrotizing Pancreatitis
}

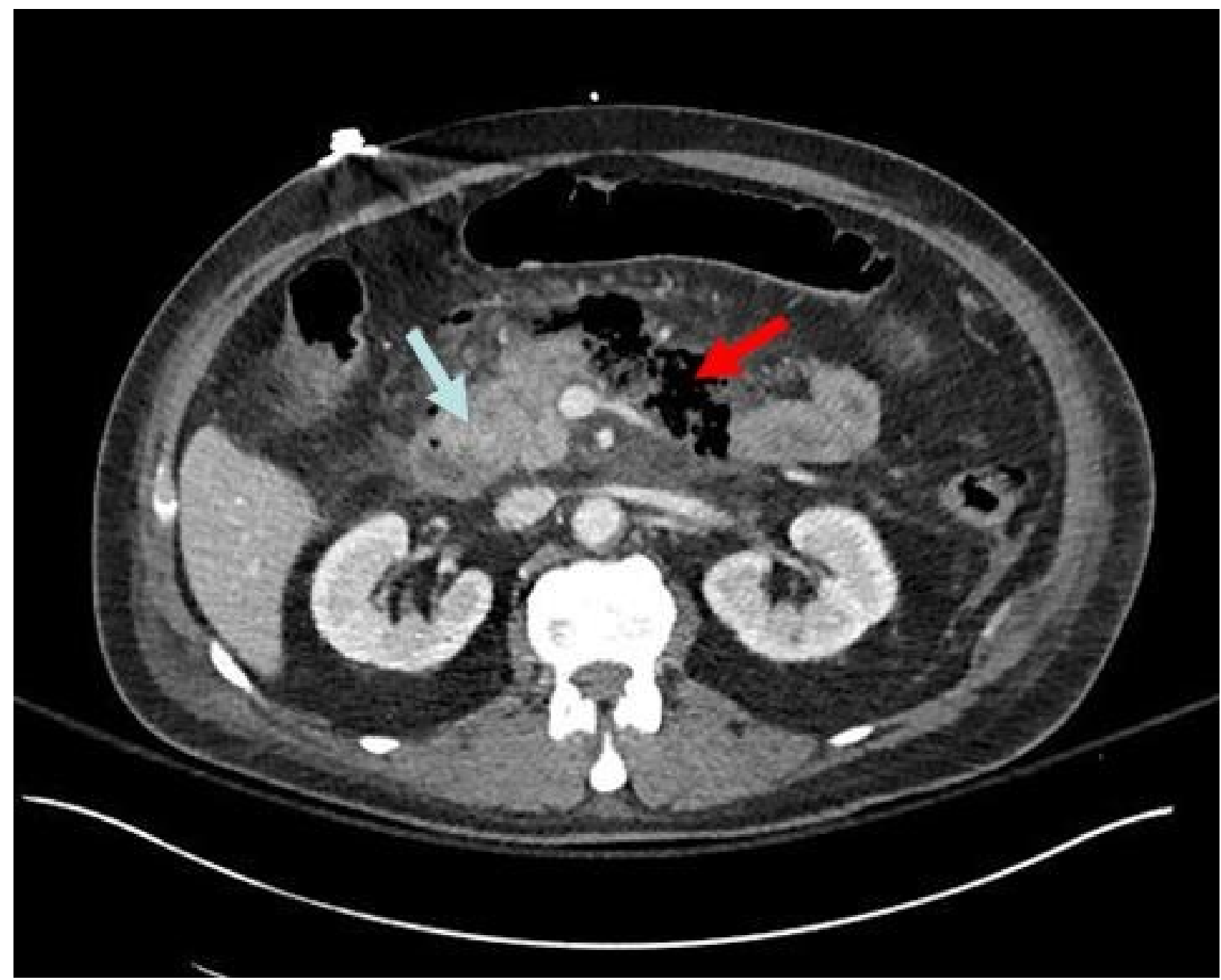

Figure 1. Contrast-enhanced CT of the abdomen and pelvis demonstrates innumerable foci of gas adjacent to the pancreatic head/body junction (red arrow) with marked inflammation of the pancreatic head (blue arrow). These findings are consistent with necrotizing pancreatitis.

A 60-year-old man with a past medical history significant for coronary artery disease status post percutaneous coronary intervention was admitted to Banner University Medical Center for acute pancreatitis complicated by a pericardial effusion requiring pericardiocentesis. The following day, the patient developed severe shortness of breath requiring increasing amounts of supplemental oxygen. The patient was emergently transferred to ICU for noninvasive bilevel positive airway pressure ventilation, but he subsequently required intubation. Throughout his worsening condition, he denied any abdominal pain, only relaying ongoing substernal chest pain. His troponins, however, remained negative and echocardiography failed to show any reaccumulation of the pericardial effusion. 
CT scan of the chest failed to show any pulmonary embolism. But, CT abdomen displayed acute pancreatitis complicated by peripancreatic gas consistent with necrotizing pancreatitis (Figure 1). Emergent laparotomy was completed. There were no signs of stomach or duodenal perforation. Purulent fluid was removed from the lesser sac and an irrigating stump was placed.

Hem Desai MD' ${ }^{1}$ Tammer Elani MD ${ }^{1}$, Nour Alhoda Parsa $M^{1}$ and Kareem Ahmad MD 2 ${ }^{1}$ Department of Internal Medicine and ${ }^{2}$ Division of Pulmonary, Allergy, Critical Care and Sleep Medicine University of Arizona

Tucson, AZ

\section{Reference}

1. Thoeni RF. The revised Atlanta classification of acute pancreatitis: Its importance for the radiologist and its effect on treatment. Radiology. 2012;262(3):751-64. [CrossRef] [PubMed] 